\title{
A GENERAL THEOREM FOR DECOMPOSITION OF LINEAR RANDOM PROCESSES
}

\author{
D. J. HEBERT, JR.
}

Abstract. Let $E$ and $F$ be locally convex spaces in duality and let $f$ be a linear random process indexed by $F$ such that the corresponding cylindrical measure is a Radon measure. It is shown without any assumptions of metrizability or countability that there is an equivalent process with continuous linear trajectories.

1. Introduction. A fundamental theorem in the theory of generalized random processes and Radonifying maps may be stated roughly as follows: If $f$ is a linear random process indexed by the dual $F$ of a locally convex space $E$ whose compact subsets are metrizable, and if the cylinder measure induced by $f$ is a Radon measure on $E$, then there is a measurable map $g$ with values in $E$ which decomposes $f$, i.e., $\langle g(t), y\rangle=[f(y)](t)$ a.e. for each $y$ in $F$. As noted by L. Schwartz in [4, (XIII. 5)], the theory of Radonifying maps often introduces spaces in which compact sets are not metrizable. It is desirable to find a more general version of this theorem which does not assume metrizability of compact subsets. The purpose of this article is to establish such a result.

2. Preliminaries. Let $E$ and $F$ be real locally convex Hausdorff linear topological spaces in duality. Let $I$ denote the collection of all finite (ordered) linearly independent subsets of $F$. If $a$ is in $I$ then $F_{a}$ is the linear span of $a$, and $a \leqq b$ means $F_{a}$ is contained in $F_{b}$. For each $a$ in $I$ there is a natural mapping $p_{a}$ of $E$ onto the dual $E_{a}$ of $F_{a}$; for $x$ in $E, p_{a}(x)$ is the restriction of $x$, considered as a linear functional, to $F_{a}$. If $a \leqq b$ there is a similar natural map $p_{a b}$ of $E_{b}$ onto $E_{a}$. If $p_{a a}$ is the identity map on $E_{a}$, then since for $a \leqq b \leqq c, p_{a c}=p_{a b} \circ p_{b c}$ and since the maps $p_{a b}$ are continuous, linear, and surjective, the family $\left(E_{a}, p_{a b}\right)$ forms a projective system of linear topological spaces. The maps $p_{a}$ form a consistent family of continuous linear maps since $p_{a}=p_{a b} \circ p_{b}$ for $a \leqq b$. Since $F$ separates the points of $E$, the maps $p_{a}$ separate the points of $E$; i.e., if $x \neq y$ in $E$ then for some $a, p_{a}(x) \neq p_{a}(y)$.

Received by the editors February 4, 1972 and, in revised form, August 4, 1972.

AMS (MOS) subject classifications (1970). Primary 28A40, 46G15, 46A05, 46F05, 60G20.

Key words and phrases. Generalized processes, cylindrical measures, lifting, decomposition.

(C) American Mathematical Society 1973 
If $(\Omega, \mathscr{F}, \mu)$ is a finite measure space, then $L^{0}=L^{0}(\Omega, \mathscr{F}, \mu)$ denotes the space of $\mu$-equivalence classes of $\mathscr{F}$-measurable real valued functions. A version of a member $l$ of $L^{0}$ is an element of the equivalence class of $l$. A linear mapping of $F$ into $L^{0}$ is called a linear process indexed by $F$. A version of a linear process $f$ is a linear mapping $g$ of $F$ into the space of all measurable functions such that for each $y$ in $F, g(y) \in f(y)$. A version $g$ of a linear process $f$ has continuous linear trajectories if for almost all $t$ in $\Omega$, $[g(\cdot)](t)$ is a continuous linear function on $F$. If a version $g$ of $f$ has continuous linear trajectories, then the function $h$ of $\Omega$ into $E$ defined by $\langle h(t), y\rangle=[g(y)](t)$ is called a decomposition of $f$ into $E$.

For each $a$ in $I$ let $\mathscr{B}_{a}$ be the Borel $\sigma$-field of $E_{a}$, and define $\mathscr{C}$ to be the collection of all subsets of $E$ of the form $p_{a}^{-1}(B)$ for $B$ in $\mathscr{B}_{a}$, and $a$ in $I$. Members of $\mathscr{C}$ are called cylinder sets of $E$. Let $f$ be a linear process indexed by $F$, mapping $F$ into $L^{0}(\Omega, \mathscr{F}, \mu)$. For each $a$ in $I$ there is a measurable map $f_{a}$ of $\Omega$ into $E_{a}$ defined as follows: If $a=\left\{y_{1}, \cdots, y_{n}\right\}$, let $f_{k}$ be a version of $f\left(y_{k}\right)$ and define $f_{a}(t)=\left(f_{1}(t), \cdots, f_{n}(t)\right)$ in $E_{a}$. If $a=\{y\}$ we write $f_{y}$ instead of $f_{a}$. If $y$ is in $F_{a}$ and $y=\sum b_{k} y_{k}$ then $\left\langle f_{a}(t), y\right\rangle=$ $\sum b_{k} f_{k}(t)=[f(y)](t)$, a.e. $(\mu)$. For each $a$, the Borel measure $\lambda_{a}=f_{a}(\mu)$ defined by $\lambda_{a}(B)=\mu\left(f_{a}^{-1}(B)\right)$ is independent of the version of $f_{a}$. If $a \leqq b$ then $p_{a b} f_{b}(\omega)=f_{a}(\omega)$ a.e. and $p_{a b}\left(\lambda_{b}\right)=\lambda_{a}$. The finitely additive function $\lambda$ defined on $\mathscr{C}$ by the formula $\lambda\left(p_{a}^{-1}(B)\right)=\lambda_{a}(B)$ is called the cylinder measure induced by $f$. Linear processes $f$ and $g$ indexed by $F$ with values in possibly different spaces $L^{0}$ are said to be equivalent if they determine the same cylinder measure.

The statement that $A$ is contained in $B$, a.e. $(\mu)$, means that $I_{A} \leqq I_{B}$, a.e. $(\mu)$, where $I_{A}, I_{B}$ are the indicator (characteristic) functions for $A$ and $B$ respectively.

Let $F^{*}$ denote the algebraic dual of $F$. For each $a$ in $I$ there is a natural map $\pi_{a}$ of $F^{*}$ onto $E_{a}$ defined by $\left\langle\pi_{a}(x), y\right\rangle=\langle x, y\rangle$ for $x$ in $F^{*}$ and $y$ in $F_{a}$. Let $\mathscr{C}^{*}$ denote the collection of cylinder sets of $F^{*}$ (sets of the form $\pi_{a}^{-1}(B)$, $B$ in $\mathscr{B}_{a}, a$ in $I$ ). If $\lambda$ is a cylinder measure on $E$ determined by a linear process $f$ then there is a cylinder measure $\lambda^{\prime}$ on $F^{*}$ defined by $\lambda^{\prime}\left(\pi_{a}^{-1}(B)\right)=$ $\lambda_{a}(B)=\mu\left(f_{a}^{-1}(B)\right)=\lambda\left(p_{a}^{-1}(B)\right)$. By a theorem of Bochner, (cf. Badrikian [1]), the cylinder measure $\lambda^{\prime}$ extends to a (countably additive) measure on $\sigma_{\mathscr{C}}^{*}$, the $\sigma$-algebra generated by $\mathscr{C}^{*}$. Suppose $\lambda$ extends to a bounded Radon measure on $E$, i.e., a finite measure on the Borel sets of $E$ such that $\lambda(B)=\sup \{\lambda(C): C$ is a compact subset of $B\}$ for each Borel set $B$. The $\lambda^{\prime}$-outer measure of $E$ in $F^{*}$ is then $\lambda(E)$, for if $E$ is contained in the union of a sequence of sets $\pi_{a_{n}}^{-1}\left(A_{n}\right)$, where $A_{n}$ is in $\mathscr{B}_{a_{n}}$, then $E$ is contained in the union of the sequence $\left\{p_{a_{n}}^{-1}\left(A_{n}\right)\right\}$; hence

$$
\lambda(E) \leqq \lambda\left(\bigcup p_{a_{n}}^{-1}\left(A_{n}\right)\right) \leqq \sum \lambda\left(p_{a_{n}}^{-1}\left(A_{n}\right)\right)=\sum \lambda^{\prime}\left(\pi_{a_{n}}^{-1}\left(A_{n}\right)\right) .
$$


3. The shadow of a compact subset of $E$. In this section, $T$ is a Hausdorff topological space, $\mu$ is a bounded Radon measure on $T$ and $f$ is a linear process indexed by $F$, mapping $F$ into $L^{0}(T, \mu)$. Suppose that $K$ is a compact subset of $E$. Let $K_{a}=p_{a}(K)$ and assume that $\inf \left\{\lambda_{a}\left(K_{a}\right): a \in I\right\}=c>0$, where $\lambda$ is the cylinder measure induced by $f$ and $\lambda_{a}=p_{a}(\lambda)$ as before. Restricting the maps $p_{a}$ and $p_{a b}$ to $K$ and $K_{b}$, the family $\left(K_{a}, p_{a b}\right)$ forms a projective system of topological spaces and the maps $p_{a}$ form a consistent family of continuous maps which separate the points of $K$. If $\gamma_{a}$ is the restriction of $\lambda_{a}$ to $K$ then the family of measures $\gamma_{a}$ forms a subprojective system, i.e., $p_{a b}\left(\gamma_{b}\right) \leqq \gamma_{a}$. A fundamental proposition in Bourbaki [2, p. 51] states that there is a unique bounded Radon measure $\gamma$ on $K$ such that $\gamma(C)=\inf \left\{\gamma_{a}\left(p_{a}(C)\right): a \in I\right\}$ for each compact subset $C$ of $K$. If $\gamma$ extends to a Radon measure on $E$ then $\gamma$ is the restriction of $\lambda$ to $K[2$, p. 52].

Consider the projective system $\left(T_{a}, i_{a b}\right)$ where $T_{a}=T$ and $i_{a b}$ is the identity map. Let $M_{a}=f_{a}^{-1}\left(K_{a}\right)$ and let $v_{a}$ be the restriction of $\mu$ to $M_{a}$. Note then that $f_{a}\left(v_{a}\right)=\gamma_{a}$. If $a \leqq b$ then since $f_{a}=p_{a b} \circ f_{b}$, a.e. $(\mu), M_{b}$ is contained in $M_{a}$, a.e. $(\mu)$. Since for each Borel set $A$ of $T, i_{a b}\left(v_{b}\right)(A)=$ $v_{b}(A)=\mu\left(A \cap M_{b}\right) \leqq \mu\left(A \cap M_{a}\right)=v_{a}(A)$, the family of measures $v_{a}$ forms a subprojective system. It follows as before that there is a unique bounded Radon measure $v$ on $T$ such that $v(C)=\inf \left\{v_{a}(C): a \in I\right\}$ for each compact subset $C$ of $T$. For each $a, v_{a}$ is absolutely continuous with respect to $\mu$, hence $v$ is absolutely continuous with respect to $\mu$. Let $r$ be the RadonNikodym derivative of $v$ with respect to $\mu$. The support $M$ of $r$ is called the shadow of $K$ in $T(M=\{t: r(t) \neq 0\})$. The following lemmas give the main properties of $M$.

\subsection{Lemma. $M$ is contained in $M_{a}$, a.e. $(\mu)$.}

Proof. It suffices to show that $r=0$, a.e. $(\mu)$, on the complement of $M_{a}$. If $Q$ is the complement of $M_{a}$, then $\int_{Q} r d \mu=\nu Q \leqq v_{a}(Q)=0$. Since $r$ is nonnegative, $r=0$, a.e. $(\mu)$, on $Q$.

3.2. Lemma. $\mu(M)=\inf \left\{\mu\left(M_{a}\right): a \in I\right\}$. Hence

$$
v(T)=\mu(M)=\inf \left\{\gamma_{a}\left(K_{a}\right): a \in I\right\}=\inf \left\{\lambda_{a}\left(K_{a}\right): a \in I\right\}=c .
$$

If $\lambda$ extends to a Radon measure on $E$, then $\mu(M)=\lambda(K)$.

Proof. For $\varepsilon>0$ there is a compact subset $C$ of $T$ such that $\nu(C) \geqq$ $v(T)-\varepsilon$. For each $a, \mu\left(M_{a}\right) \geqq \mu\left(M_{a} \cap C\right)=v_{a}(C) \geqq v(C) \geqq v(T)-\varepsilon$. So $c=$ $\inf \left\{\mu\left(M_{a}\right): a \in I\right\} \geqq v(T)$. Now for $\delta>0$ choose a compact set $D$ such that $v(D) \geqq v(T)-\delta$. Since $\mu\left(M_{a}\right)=\lambda_{a}\left(K_{a}\right) \geqq c$ for each $a, v_{a}(D)=\mu\left(D \cap M_{a}\right) \geqq$ $c-\delta$ for each $a$. Hence $v(D)=\inf \left\{v_{a}(D)=a \in A\right\} \geqq c-\delta$. This implies $v(T) \geqq c-\delta$. 
3.3. LEMMA. If $\lambda$ extends to a bounded Radon measure on $E$, then for each $a$ in $I$ and for each $A$ in $\mathscr{B}_{a}$, the following properties hold:

(i) $\lambda\left(p_{a}^{-1}(A) \cap K\right)=\inf \left\{\lambda\left(p_{a}^{-1}(A) \cap p_{b}^{-1}\left(K_{b}\right)\right): b \in I\right\}$;

(ii) $\mu\left(f_{a}^{-1}(A) \cap M\right)=\inf \left\{\mu\left(f_{a}^{-1}(A) \cap M_{b}\right): b \in I\right\}$;

(iii) $\lambda\left(p_{a}^{-1}(A) \cap p_{b}^{-1}\left(K_{b}\right)\right)=\mu\left(f_{a}^{-1}(A) \cap M_{b}\right)$ for each $b \geqq a$;

(iv) $\lambda\left(p_{a}^{-1}(A) \cap K\right)=\mu\left(f_{a}^{-1}(A) \cap M\right)$.

Proof. (i) For $\varepsilon>0$ pick $b \geqq a$ such that $\lambda\left(p_{b}^{-1}\left(K_{b}\right)\right)-\lambda(K)<\varepsilon$; then $\lambda\left(p_{a}^{-1}(A) \cap p_{b}^{-1}\left(K_{b}\right)\right)-\lambda\left(p_{a}^{-1}(A) \cap K\right)<\varepsilon$, since $p_{b}^{-1}\left(K_{b}\right)$ contains $K$.

(ii) For $\varepsilon>0$, Lemma 3.2 provides $b \geqq a$ such that $\mu\left(M_{b}\right)-\mu(M)<\varepsilon$; then since $M_{a}$ contains $M$ a.e., $\mu\left(f_{a}^{-1}(A) \cap M_{b}\right)-\mu\left(f_{a}^{-1}(A) \cap M\right)<\varepsilon$.

$$
\begin{aligned}
\lambda\left(p_{a}^{-1}(A) \cap p_{b}^{-1}\left(K_{b}\right)\right) & =\lambda\left(p_{b}^{-1} \circ p_{a b}^{-1}(A) \cap p_{b}^{-1}\left(K_{b}\right)\right) \\
& =\mu\left(f_{b}^{-1}\left(p_{a b}^{-1}(A) \cap K_{b}\right)\right)=\mu\left(f_{a}^{-1}(A) \cap M_{b}\right) .
\end{aligned}
$$

(iv) follows from (i), (ii), and (iii).

3.4. Lemma. The restriction of $\mu$ to $M$ is $\nu$.

Proof. If $C$ is a compact subset of $M$, then for each $a$ in $I, M_{a}$ contains $C$, a.e. $(\mu)$, by Lemma 3.1. It follows that $\nu_{a}(C)=\mu\left(C \cap M_{a}\right)=\mu(C)$, hence $v(C)=\mu(C)$.

3.5. LEMMA. If $h(y)$ is the restriction of $f(y)$ to $M$ for each $y$ in $F$, then $h$ is a linear mapping of $F$ into $L^{\infty}(M, v)$.

Proof. If $y \in F$ then $p_{y}(K)=K_{y}$ is a compact set of real numbers; hence $K_{y}$ is contained in an interval of the form $[-m, m]$. Let $A$ be the subset of $T$ on which $\left|f_{y}(t)\right|>m\left(f_{y}\right.$ is a version of $\left.f(y)\right)$. Since $f_{y}^{-1}[-m, m]$ contains $f_{y}^{-1}\left(K_{y}\right)=M_{y}$ which contains $M$, a.e. $(\mu)$, it follows that $\nu(A)=0$.

3.6. LeMMA. There is a mapping $g$ of $M$ into $F^{*}$ such that $\langle g(t), y\rangle=$ $f_{y}(t)$, a.e. $(v)$, for each $y$ in $F$.

Proof. By the lifting theorem of Ionescu-Tulcea [3], there is a linear mapping $\rho$ of $L^{\infty}(M, v)$ into $\mathscr{L}^{\infty}(M, v)$, the space of all bounded measurable functions on $T$, such that $\rho(l) \in h$ for each $l$ in $L^{\infty}(M, v)$. Let $\langle g(t), y\rangle=$ [ $\rho \circ h(y)](t)$, where $h(y)$ is the restriction of $f(y)$ to $M$, for each $y$ in $F$.

Now suppose that $K$ and $L$ are compact subsets of $E$ such that $K$ is a subset of $L$. Let $M$ and $N$ be the shadows of $K$ and $L$ respectively in $T$. Assume also that $0<c=\inf \left\{\lambda_{a}\left(K_{a}\right): a \in I\right\}$ and $0<d=\inf \left\{\lambda_{a}\left(L_{a}\right): a \in I\right\}$.

3.7. LeMma. $M$ is contained in $N$, a.e. $(\mu)$.

Proof. Let $M_{a}=f_{a}^{-1}\left(K_{a}\right)$ and let $N_{a}=f_{a}^{-1}\left(L_{a}\right)$ for each $a$. There are increasing sequences $\left(a_{k}\right)$ and $\left(b_{k}\right)$ in $I$ such that $c=\inf \left\{\lambda_{a_{k}}\left(K_{a_{k}}\right)\right\}=$ $\inf \left\{\mu\left(M_{a_{k}}\right)\right\}$ and $d=\inf \left\{\mu\left(N_{b_{k}}\right)\right\}$. For each $k$ choose $e_{k} \geqq a_{k}, b_{k}, e_{k-1}$. Now 
since $\mu\left(M_{a_{k}}\right) \geqq \mu\left(M_{e_{k}}\right)$ and $M_{e_{k-1}}$ contains $M_{e_{k}}$, a.e. $(\mu), \inf \left\{\mu\left(M_{e_{k}}\right)\right\}=c=$ $\mu\left(\bigcap M_{e_{k}}\right)$. Since $M_{a}$ contains $M$, a.e. $(\mu)$, for each $a, \bigcap M_{e_{k}}$ contains $M$, a.e. $(\mu)$, and since $\mu(M)=c=\mu\left(\cap M_{e_{k}}\right)$, by Lemma 3.2, it follows that $M=\bigcap M_{e_{k}}$, a.e. $(\mu)$. By similar reasoning $N=\bigcap N_{e_{k}}$, a.e. $(\mu)$. Since for each $k, N_{e_{k}}$ contains $M_{e_{k}}$, a.e. $(\mu), N$ contains $M$, a.e. $(\mu)$.

\section{Statement and proof of the theorem.}

THEOREM. If $E$ and $F$ are locally convex spaces in duality and $f$ is a linear process mapping $F$ into $L^{0}(T, \mu)$ where $T$ is a Hausdorff space and $\mu$ is a bounded Radon measure on $T$, and if the cylinder measure induced by $f$ has an extension which is a Radon measure on $E$, then there is a subset $W$ of $T$, a sub- $\sigma$-algebra $\mathscr{F}$ of the Borel sets of $W$, a measure $v$ on $\mathscr{F}$, and a linear process $h$ mapping $F$ into $L^{0}(W, \mathscr{F}, v)$ such that $h$ is equivalent to $f$ and $h$ has a version with continuous linear trajectories.

Proof. Choose an increasing sequence $\left(K^{n}\right)$ of compact subsets of $E$ such that, for each $n, \lambda\left(K^{n}\right)=c_{n} \geqq \lambda(E)-1 / n$. Let $M^{n}$ be the shadow of $K^{n}$ in $T$, let $\mu^{n}$ be the restriction of $\mu$ to $M^{n}$ and let $g^{n}$ be a mapping of $M^{n}$ into $F^{*}$ such that $\left\langle g^{n}(t), y\right\rangle=f_{y}(t)$, a.e. $\left(\mu^{n}\right)$, as in Lemma 3.6. Let $N^{n}$ be the union of the sets $M^{k}$ for $k \leqq n$ and define $g(t)=g^{n}(t)$ for $t$ in $N^{n}-N^{n-1}$. If $Y$ is the union of the sets $M^{n}$, then $\langle g(t), y\rangle=f_{y}(t)$, a.e. $(\mu)$, on the set $Y$. Since $\lambda(E)=\sup \lambda\left(K^{n}\right)=\sup \mu\left(M^{n}\right)$ by Lemma 3.2, $\mu(Y)=\lambda(E)$. Let $\mu_{0}$ be the restriction of $\mu$ to the Borel sets of $Y$ of the form $g^{-1}(S)$ for $S$ in $\sigma_{\mathscr{C}}^{*}$. For each Borel set $A$ of $E_{a}$,

$$
\begin{aligned}
\mu_{0}\left(g^{-1} \circ \pi_{a}^{-1}(A)\right) & =\mu\left[Y \cap g^{-1} \circ \pi^{-1}(A)\right]=\mu\left[\bigcup\left(N^{n}-N^{n-1}\right) \cap f_{a}^{-1}(A)\right] \\
& =\mu\left[Y \cap f_{a}^{-1}(A)\right]=\sup \mu\left(M^{n} \cap f_{a}^{-1}(A)\right) \\
& =\sup \lambda\left(K^{n} \cap p_{a}^{-1}(A)\right)=\lambda\left(p_{a}^{-1}(A)\right)=\lambda^{\prime}\left(\pi_{a}^{-1}(A)\right) .
\end{aligned}
$$

Let $W=g^{-1}(E)$. If $\mu_{0}^{*}$ is the outer measure induced by $\mu_{0}$ on subsets of $Y$ then since the $\lambda^{\prime}$-outer measure of $E$ is $\lambda(E)$,

$$
\begin{aligned}
\mu_{0}^{*}(W) & =\inf \left\{\mu_{0}\left(g^{-1}(A)\right): A \in \sigma \mathscr{C}^{*}, W \subseteq g^{-1}(A)\right\} \\
& =\inf \left\{\lambda^{\prime}(A): A \in \sigma \mathscr{C}^{*}, E \subseteq A\right\}=\lambda(E)=\mu_{0}(Y) .
\end{aligned}
$$

Let $\mathscr{F}$ be the $\sigma$-field consisting of sets of the form $g^{-1}(A) \cap W$ for $A$ in $\sigma \mathscr{C}^{*}$. Define $v\left(g^{-1}(A) \cap W\right)=\mu_{0}\left(g^{-1}(A)\right)=\lambda^{\prime}(A)$ for each $A$ in $\sigma \mathscr{C}^{*}$; then $\nu$ is a measure on $\mathscr{F}$. If $[h y](t)=p_{y} \circ g(t)=\langle g(t), y\rangle$ for each $y$ in $F$ and $t$ in $W$, then $h$ is a (version with continuous linear trajectories of a) linear process mapping $F$ into $L^{0}(W, \mathscr{F}, v)$. The cylinder measure induced by $h$ is $\lambda$ so that $h$ and $f$ are equivalent processes.

REMARK. The theorem mentioned in the introduction is contained in the above. If there is a countable cofinal family of finite dimensional subspaces of $F$, in particular, if compact subsets of $E$ are metrizable, then the 
compact sets $K^{n}$ in the preceding proof are members of $\sigma \mathscr{C}^{*}\left(K^{n}=\right.$ $\bigcap \pi_{a}^{-1}\left(K_{a}^{n}\right)$ can be expressed as a countable intersection). It follows that $Y=g^{-1}\left(\bigcup K^{n}\right)$ a.e.; hence $g$ can be redefined as a version of $f$ with continuous linear trajectories.

\section{REFERENCES}

1. A. Badrikian, Séminaire sur les fonctions aléatoires linéaires et les mésures cylindriques, Lecture Notes in Math., vol. 139, Springer-Verlag, Berlin and New York, 1970. MR 43 \#4994.

2. N. Bourbaki, Éléments de mathématique. Fasc. XXXV. Livre VI: Intégration. Chap. 9: Intégration sur les espaces topologiques séparés, Actualités Sci. Indust., no. 1343, Hermann, Paris, 1969. MR 43 \#2183.

3. A. Ionescu Tulcea and C. Ionescu Tulcea, Topics in the theory of lifting, Ergebnisse der Mathematik, und ihrer Grenzgebiete, Band 48, Springer-Verlag, New York, 1969. MR 43 \#2185.

4. L. Schwartz, Séminaire Laurent Schwartz-applications radonifiantes, École Polytechnique, Centre de Mathématiques, Paris, 1969/70.

Department of Mathematics, University of Pittsburgh, Pittsburgh, PennsylVANIA 15213 